\title{
ON SHARP CONSTANTS IN MARCINKIEWICZ-ZYGMUND AND PLANCHEREL-POLYA INEQUALITIES
}

\author{
D. S. LUBINSKY
}

(Communicated by Walter Van Assche)

Abstract. The Plancherel-Polya inequalities assert that for $1<p<\infty$, and entire functions $f$ of exponential type at most $\pi$,

$$
A_{p} \sum_{j=-\infty}^{\infty}|f(j)|^{p} \leq \int_{-\infty}^{\infty}|f|^{p} \leq B_{p} \sum_{j=-\infty}^{\infty}|f(j)|^{p} .
$$

The Marcinkiewicz-Zygmund inequalities assert that for $n \geq 1$ and polynomials $P$ of degree $\leq n-1$,

$$
\frac{A_{p}^{\prime}}{n} \sum_{j=1}^{n}\left|P\left(e^{2 \pi i j / n}\right)\right|^{p} \leq \int_{0}^{1}\left|P\left(e^{2 \pi i t}\right)\right|^{p} d t \leq \frac{B_{p}^{\prime}}{n} \sum_{j=1}^{n}\left|P\left(e^{2 \pi i j / n}\right)\right|^{p} .
$$

We show that the sharp constants in both inequalities are the same; that is, $A_{p}=A_{p}^{\prime}$ and $B_{p}=B_{p}^{\prime}$. Moreover, the two inequalities are equivalent. We also discuss the case $p \leq 1$.

\section{INTRODUCTION}

The Plancherel-Polya inequalities [5, p. 152] assert that for $1<p<\infty$ and entire functions $f$ of exponential type at most $\pi$,

$$
A_{p} \sum_{j=-\infty}^{\infty}|f(j)|^{p} \leq \int_{-\infty}^{\infty}|f|^{p} \leq B_{p} \sum_{j=-\infty}^{\infty}|f(j)|^{p},
$$

provided either the series or integral is finite. For $0<p \leq 1$, the left-hand inequality is still true, but the right-hand inequality requires additional restrictions [1], 3], [9]. Of course, $A_{p}, B_{p}$ are independent of $f$. Moreover, a dilation of the variable yields an analogous inequality for $f$ of any given finite type. These inequalities play an important role in sampling theory and applications of Paley-Wiener spaces [5].

The Marcinkiewicz-Zygmund inequalities assert [11, Vol. II, p. 30] that for $p>1$, $n \geq 1$, and polynomials $P$ of degree $\leq n-1$,

$$
\frac{A_{p}^{\prime}}{n} \sum_{j=1}^{n}\left|P\left(e^{2 \pi i j / n}\right)\right|^{p} \leq \int_{0}^{1}\left|P\left(e^{2 \pi i t}\right)\right|^{p} d t \leq \frac{B_{p}^{\prime}}{n} \sum_{j=1}^{n}\left|P\left(e^{2 \pi i j / n}\right)\right|^{p} .
$$

Here too, $A_{p}^{\prime}$ and $B_{p}^{\prime}$ are independent of $n$ and $P$, and the left-hand inequality is also true for $0<p \leq 1$ [7]. These inequalities are useful in studying convergence

Received by the editors November 9, 2012.

2010 Mathematics Subject Classification. Primary 30D15, 30D99, 41A17, 41A55; Secondary 26D15, 26D05.

Key words and phrases. Plancherel-Polya inequalities, Marcinkiewicz-Zygmund inequalities, Entire functions, quadrature sums.

Research supported by NSF grant DMS1001182 and US-Israel BSF grant 2008399. 
of Fourier series, Lagrange interpolation, in number theory, and weighted approximation. They have been extended to many settings, and there are a great many methods to prove them [4, [7, 8].

To the best of this author's knowledge, the sharp constants in (1.1) and (1.2) are unknown, except for the case $p=2$, where of course $A_{2}=B_{2}=A_{2}^{\prime}=B_{2}^{\prime}=1$ [5. p. 150]. Throughout, we assume that $A_{p}, B_{p}, A_{p}^{\prime}, B_{p}^{\prime}$ are the sharp constants, so that $A_{p}$ and $A_{p}^{\prime}$ are as large as possible, while $B_{p}$ and $B_{p}^{\prime}$ are as small as possible. The main result of this paper is:

Theorem 1.1. For $0<p<\infty$,

$$
A_{p}=A_{p}^{\prime}
$$

and for $1<p<\infty$,

$$
B_{p}=B_{p}^{\prime} .
$$

This theorem can be seen as a further example of the long-standing connection between asymptotics for polynomials and entire functions of exponential type [2], [10. Chapters 4, 5]. Indeed, the main idea in the proof is that scaling limits transform polynomials into entire functions of exponential type. We also prove a duality inequality. Let $\sigma_{p}$ denote the norm of the Fourier partial sum projection in $L_{p}$, that is

$$
\begin{gathered}
\sigma_{p}=\sup \left\{\int_{0}^{1}\left|\sum_{j=0}^{n} c_{j} e^{2 \pi i j t}\right|^{p} d t: n \geq 1,\left\{c_{j}\right\} \subset \mathbb{C}\right. \\
\left.\quad \text { and } \int_{0}^{1}\left|\sum_{j=-\infty}^{\infty} c_{j} e^{2 \pi i j t}\right|^{p} d t \leq 1\right\} .
\end{gathered}
$$

Theorem 1.2. (a) Let $1<p<\infty, p \neq 2$, and $q=\frac{p}{p-1}$. Then

$$
\left(B_{q}^{1 / q}\right)^{-1} \leq A_{p}^{1 / p} \leq\left(B_{q}^{1 / q}\right)^{-1} \sigma_{p}^{1 / p}
$$

(b) Moreover, for all $p>1$ except $p=2$,

$$
B_{p}>1
$$

while for $0<p<\infty$ except $p=2$,

$$
A_{p}<1 \text {. }
$$

In [1, p. 101, Thm. 6.7.15], it is proven that $B_{p} \leq \frac{4}{\pi} e^{p \pi / 2}$.

\section{ProOFS}

Throughout $C, C_{1}, C_{2}, \ldots$ denote constants independent of $n, m, x, t$. We shall use the sinc kernel $S(z)=\frac{\sin \pi z}{\pi z}$, an entire function of exponential type $\pi$. We shall also use the fact that for $\lambda>1$,

$$
\lim _{m \rightarrow \infty} \frac{1}{m} \sum_{k=-\infty}^{\infty}\left|S\left(\frac{k+x}{m}\right)\right|^{\lambda}=\int_{-\infty}^{\infty}|S(t)|^{\lambda} d t,
$$


uniformly for $x \in[0,1]$. This is easily established: if $r \geq 2$,

$$
\begin{aligned}
& \frac{1}{m} \sup _{x \in[0,1]} \sum_{k:|k| \geq m r}^{\infty}\left|S\left(\frac{k+x}{m}\right)\right|^{\lambda}+\int_{|t| \geq r}|S(t)|^{\lambda} d t \\
& \quad \leq C\left(\frac{1}{m} \sum_{k:|k| \geq m r}^{\infty}\left(\frac{m}{|k|}\right)^{\lambda}+\int_{|t| \geq r} \frac{1}{|t|^{\lambda}} d t\right) \leq C r^{1-\lambda},
\end{aligned}
$$

so that the tails of both the series and the integral are uniformly small for large $r$ while $S$ is uniformly continuous in $\mathbb{R}$, then the theory of Riemann sums yields uniformly for $x \in[0,1]$,

$$
\lim _{m \rightarrow \infty} \frac{1}{m} \sum_{k:|k| \leq m r}\left|S\left(\frac{k+x}{m}\right)\right|^{\lambda}=\int_{-r}^{r}|S(t)|^{\lambda} d t .
$$

We start with:

Proof that for $p>0, A_{p} \leq A_{p}^{\prime}$. Let $n \geq 1$ and $P$ be a polynomial of degree $\leq n-1$. Choose positive integers $m, J$ such that $J p>1$ and $m>J$. Let

$$
f(z)=e^{-i \pi\left(1-\frac{1}{n}\right) z} P\left(e^{2 \pi i z / n}\right) S\left(\frac{z}{m n}\right)^{J} .
$$

It is easily seen that $f$ is entire of exponential type $\leq \pi$. Moreover,

$$
\begin{aligned}
\sum_{j=-\infty}^{\infty}|f(j)|^{p} & =\sum_{k=-\infty}^{\infty} \sum_{\ell=0}^{n-1}|f(k n+\ell)|^{p} \\
& =\sum_{\ell=0}^{n-1}\left|P\left(e^{2 \pi i \ell / n}\right)\right|^{p} \sum_{k=-\infty}^{\infty}\left|S\left(\frac{k+\ell / n}{m}\right)\right|^{J p},
\end{aligned}
$$

while

$$
\begin{aligned}
\int_{-\infty}^{\infty}|f(x)|^{p} d x & =n \int_{-\infty}^{\infty}\left|P\left(e^{2 \pi i t}\right)\right|^{p}\left|S\left(\frac{t}{m}\right)\right|^{J p} d t \\
& =n \int_{0}^{1}\left|P\left(e^{2 \pi i s}\right)\right|^{p} \sum_{k=-\infty}^{\infty}\left|S\left(\frac{k+s}{m}\right)\right|^{J p} d s
\end{aligned}
$$

We can then recast the left inequality in (1.1) for this function $f$ as

$$
\begin{aligned}
A_{p} & \frac{1}{n} \sum_{\ell=0}^{n-1}\left|P\left(e^{2 \pi i \ell / n}\right)\right|^{p}\left(\frac{1}{m} \sum_{k=-\infty}^{\infty}\left|S\left(\frac{k+\ell / n}{m}\right)\right|^{J p}\right) \\
\leq & \int_{0}^{1}\left|P\left(e^{2 \pi i s}\right)\right|^{p}\left(\frac{1}{m} \sum_{k=-\infty}^{\infty}\left|S\left(\frac{k+s}{m}\right)\right|^{J p}\right) d s .
\end{aligned}
$$

We now let $m \rightarrow \infty$ and use the uniform convergence in (2.1) to obtain

$$
A_{p} \frac{1}{n} \sum_{\ell=0}^{n-1}\left|P\left(e^{2 \pi i \ell / n}\right)\right|^{p} \leq \int_{0}^{1}\left|P\left(e^{2 \pi i s}\right)\right|^{p} d s .
$$

Since this holds for any $n \geq 1$ and any polynomial $P$ of degree $\leq n-1$, it follows that $A_{p}^{\prime} \geq A_{p}$. 
Proof that for $p>1, B_{p} \geq B_{p}^{\prime}$. Let $P$ have degree $\leq n-1$. We proceed as above, but use the right inequality in (1.1), to obtain

$$
\begin{aligned}
& \int_{0}^{1}\left|P\left(e^{2 \pi i s}\right)\right|^{p}\left(\frac{1}{m} \sum_{k=-\infty}^{\infty}\left|S\left(\frac{k+s}{m}\right)\right|^{J p}\right) d s \\
& \quad \leq B_{p} \frac{1}{n} \sum_{\ell=0}^{n-1}\left|P\left(e^{2 \pi i \ell / n}\right)\right|^{p}\left(\frac{1}{m} \sum_{k=-\infty}^{\infty}\left|S\left(\frac{k+\ell / n}{m}\right)\right|^{J p}\right) .
\end{aligned}
$$

Letting $m \rightarrow \infty$ yields

$$
\int_{0}^{1}\left|P\left(e^{2 \pi i s}\right)\right|^{p} d s \leq B_{p} \frac{1}{n} \sum_{\ell=0}^{n-1}\left|P\left(e^{2 \pi i \ell / n}\right)\right|^{p} .
$$

As above, it follows that $B_{p} \geq B_{p}^{\prime}$.

The converse inequalities are more difficult:

Proof that for $p>1, B_{p} \leq B_{p}^{\prime}$ and hence $B_{p}=B_{p}^{\prime}$. Let $f$ be an entire function of exponential type $\leq \pi$, for which the integral in (1.1) is convergent. Then $f$ admits the sampling series expansion [5, p. 152]

$$
f(z)=\sum_{k=-\infty}^{\infty} f(k) S(z-k) .
$$

It converges uniformly in compact subsets of the plane. Moreover,

$$
\lim _{L \rightarrow \infty} \int_{-\infty}^{\infty}\left|f(x)-\sum_{|k| \leq L} f(k) S(x-k)\right|^{p} d x=0 .
$$

For $|j| \leq[n / 2]$, let

$$
\ell_{j n}(z)=\frac{1}{n} \frac{z^{n}-1}{z e^{-2 \pi i j / n}-1}
$$

denote the $j$ th fundamental polynomial of Lagrange interpolation at the $n$th roots of unity. If $n$ is even, we consider only $j=n / 2$, not $j=-n / 2$. Let us fix $L \geq 1$, and for $n>2 L$, define

$$
P_{n}(z)=\sum_{|j| \leq L} f(j)(-1)^{j} \ell_{j n}(z),
$$

a polynomial of degree $\leq n-1$. Then

$$
\sum_{j=1}^{n}\left|P_{n}\left(e^{2 \pi i j / n}\right)\right|^{p}=\sum_{|j| \leq L}|f(j)|^{p} .
$$

A straightforward calculation shows that for fixed $j$,

$$
\lim _{n \rightarrow \infty} \ell_{j n}\left(e^{2 \pi i t / n}\right)=e^{i \pi t}(-1)^{j} S(t-j),
$$

uniformly for $t$ in compact sets. Moreover, for $\frac{1}{3} \geq|s| \geq 2|j| / n$,

$$
\left|\ell_{j n}\left(e^{2 \pi i s}\right)\right| \leq \frac{1}{n|\sin \pi(s-j / n)|} \leq \frac{1}{2 n|s-j / n|} \leq \frac{1}{n|s|} .
$$


Thus, uniformly for $t$ in compact sets,

$$
\lim _{n \rightarrow \infty} P_{n}\left(e^{2 \pi i t / n}\right)=e^{i \pi t} \sum_{|k| \leq L} f(k) S(t-k),
$$

while for $|s| \geq 2 L / n$,

$$
\left|P_{n}\left(e^{2 \pi i s}\right)\right| \leq \frac{1}{n|s|} \sum_{|k| \leq L}|f(k)|=: \frac{C_{L}}{n|s|} .
$$

Then, given $r>2 L$,

$$
\begin{aligned}
& n \int_{0}^{1}\left|P_{n}\left(e^{2 \pi i s}\right)\right|^{p} d s=n \int_{-1 / 2}^{1 / 2}\left|P_{n}\left(e^{2 \pi i s}\right)\right|^{p} d s \\
& \quad=\int_{-r}^{r}\left|P_{n}\left(e^{2 \pi i t / n}\right)\right|^{p} d t+O\left(C_{L}^{p} n \int_{|s| \geq r / n} \frac{d s}{(n|s|)^{p}}\right) \\
& \quad=\int_{-r}^{r}\left|\sum_{|k| \leq L} f(k) S(t-k)\right|^{p} d t+O\left(C_{L}^{p} r^{1-p}\right) .
\end{aligned}
$$

Using this and (2.5), we may now recast the right-hand inequality in (1.2) as

$$
\int_{-r}^{r}\left|\sum_{|k| \leq L} f(k) S(t-k)\right|^{p} d t+O\left(C_{L}^{p} r^{1-p}\right) \leq B_{p}^{\prime} \sum_{|j| \leq L}|f(j)|^{p} .
$$

Letting $r \rightarrow \infty$ gives

$$
\int_{-\infty}^{\infty}\left|\sum_{|k| \leq L} f(k) S(t-k)\right|^{p} d t \leq B_{p}^{\prime} \sum_{|j| \leq L}|f(j)|^{p} .
$$

We may now let $L \rightarrow \infty$, and use (2.3) to deduce

$$
\int_{-\infty}^{\infty}|f(t)|^{p} d t \leq B_{p}^{\prime} \sum_{j=-\infty}^{\infty}|f(j)|^{p} .
$$

As $f$ was an arbitrary entire function of exponential type $\leq \pi$, we deduce that $B_{p} \leq B_{p}^{\prime}$. Together with the previous proof, this shows that $B_{p}=B_{p}^{\prime}$.

Proof that for $p>1, A_{p} \geq A_{p}^{\prime}$ and hence $A_{p}=A_{p}^{\prime}$. Here, we proceed as above, but use the left-hand inequality in (1.2), leading to

$$
A_{p}^{\prime} \sum_{|j| \leq L}|f(j)|^{p} \leq \int_{-r}^{r}\left|\sum_{|k| \leq L} f(k) S(t-k)\right|^{p} d t+O\left(C_{L}^{p} r^{1-p}\right) .
$$

Letting $r \rightarrow \infty$ gives for $M \leq L$

$$
A_{p}^{\prime} \sum_{|j| \leq M}|f(j)|^{p} \leq \int_{-\infty}^{\infty}\left|\sum_{|k| \leq L} f(k) S(t-k)\right|^{p} d t
$$


We now let $L \rightarrow \infty$ in the right-hand side, and use (2.3), and then finally let $M \rightarrow \infty$ in the left-hand side, giving

$$
A_{p}^{\prime} \sum_{j=-\infty}^{\infty}|f(j)|^{p} \leq \int_{-\infty}^{\infty}|f(t)|^{p} d t
$$

As this holds for any such $f$, we obtain $A_{p} \geq A_{p}^{\prime}$. The converse inequality $A_{p} \leq A_{p}^{\prime}$ was proved above.

The case $p \leq 1$ is more difficult:

Proof that for $0<p \leq 1, A_{p} \leq A_{p}^{\prime}$ and hence $A_{p}=A_{p}^{\prime}$. Let $f$ be entire of exponential type $\leq \pi$, with the integral in (1.1) finite. We note that the left-hand inequality in (1.1) (which is valid even for $p \leq 1$ ), shows that $\sup _{j}|f(j)|<\infty$, and hence

$$
\sum_{j=-\infty}^{\infty}|f(j)|^{\lambda}<\infty \text { for all } \lambda>p .
$$

In particular, $f$ satisfies (1.1) with $p=2$, and consequently, we still have the sampling series expansion (2.2). Choose a positive integer $J$ such that $J p \geq 2$, and let $\varepsilon \in(0,1 / 2)$. Let $L>1$,

$$
U_{k}(z)=\frac{1}{k} \frac{z^{k}-1}{z-1}
$$

and with $[x]$ denoting the greatest integer $\leq x$, let

$$
P_{n}(z)=\left(\sum_{|j| \leq L} f(j)(-1)^{j} \ell_{j, n-[\varepsilon n]}(z)\right) U_{\left[\frac{\varepsilon}{J} n\right]}(z)^{J},
$$

a polynomial of degree $\leq n-1$. A straightforward calculation shows for as $n \rightarrow \infty$,

$$
\ell_{j, n-[\varepsilon n]}\left(e^{2 \pi i t / n}\right)=(-1)^{j} e^{i \pi t(1-\varepsilon)} S((1-\varepsilon) t-j)+o(1),
$$

uniformly for $t$ in compact sets and any fixed $j$ (cf. [6, Lemma 2.2]). In a similar way, uniformly for $t$ in compact sets,

$$
U_{\left[\frac{\varepsilon}{J} n\right]}\left(e^{2 \pi i t / n}\right)=e^{i \pi \frac{\varepsilon t}{J}} S\left(\frac{\varepsilon t}{J}\right)+o(1),
$$

so uniformly for such $t$, as $n \rightarrow \infty$,

$$
\begin{aligned}
\left|P_{n}\left(e^{2 \pi i t / n}\right)\right| & =\left|e^{i \pi t}\left(\sum_{|j| \leq L} f(j) S((1-\varepsilon) t-j)\right) S\left(\frac{\varepsilon t}{J}\right)^{J}+o(1)\right| \\
& \leq\left|\sum_{|j| \leq L} f(j) S((1-\varepsilon) t-j)\right|\left|S\left(\frac{\varepsilon t}{J}\right)^{J}\right|+o(1) \\
& \leq|f(t(1-\varepsilon))|+O\left(\sum_{|j|>L}|f(j)|\right) \min \left\{1, \frac{1}{|t|}\right\}^{J}+o(1),
\end{aligned}
$$


recall that $|S(s)| \leq \min \left\{1, \frac{1}{\pi|s|}\right\}$, and (2.7). Then for fixed $r>4 L$, we obtain, as $n \rightarrow \infty$,

$$
n \int_{-r / n}^{r / n}\left|P_{n}\left(e^{2 \pi i s}\right)\right|^{p} d s \leq \frac{1}{1-\varepsilon} \int_{-\infty}^{\infty}|f(t)|^{p} d t+C\left(\sum_{|j|>L}|f(j)|\right)^{p}+o(1) .
$$

Here, as $J p>1, C$ is independent of $r, n, L$ but does depend on $\varepsilon$ and $J$. Next, for $\frac{1}{3} \geq|s| \geq r / n,(2.6)$ gives

$$
\left|P_{n}\left(e^{2 \pi i s}\right)\right| \leq\left(\frac{C}{n|s|} \sum_{|j| \leq L}|f(j)|\right)\left(\frac{1}{\frac{\varepsilon}{J} n|s|}\right)^{J} \leq \frac{C}{r}\left(\frac{1}{n|s|}\right)^{J}
$$

where $C$ is independent of $L, s, r, n$. Then

$$
n \int_{r / n \leq|s| \leq 1 / 2}\left|P_{n}\left(e^{2 \pi i s}\right)\right|^{p} d s \leq \frac{C n}{r^{p}} \int_{r / n \leq|s| \leq 1 / 2}\left(\frac{1}{n|s|}\right)^{J p} d s \leq C r^{-p+1-J p} .
$$

Thus, as $n \rightarrow \infty$,

$$
\begin{aligned}
& n \int_{-1 / 2}^{1 / 2}\left|P_{n}\left(e^{2 \pi i s}\right)\right|^{p} d s \\
& \quad \leq \frac{1}{1-\varepsilon} \int_{-\infty}^{\infty}|f(t)|^{p} d t+C\left(\sum_{|j| \geq L}|f(j)|\right)^{p}+C r^{-p+1-J p}+o(1) .
\end{aligned}
$$

Here $C$ is independent of $r, n, L$. Next, for each fixed $k$, as $n \rightarrow \infty$,

$$
\left|P_{n}\left(e^{2 \pi i k / n}\right)\right|=\left|\sum_{|j| \leq L} f(j) S((1-\varepsilon) k-j)\right|\left|S\left(\frac{\varepsilon k}{J}\right)\right|^{J}+o(1) .
$$

The left-hand inequality in (1.2) gives, for any fixed $L \geq M \geq 1$, as $n \rightarrow \infty$,

$$
\begin{aligned}
& A_{p}^{\prime} \sum_{|k| \leq M}\left|\sum_{|j| \leq L} f(j) S((1-\varepsilon) k-j)\right|^{p}\left|S\left(\frac{\varepsilon k}{J}\right)\right|^{J p} \\
& \quad \leq \frac{1}{1-\varepsilon} \int_{-\infty}^{\infty}|f(t)|^{p} d t+C\left(\sum_{|j| \geq L}|f(j)|\right)^{p}+C r^{-p+1-J p} .
\end{aligned}
$$

Here $C$ is independent of $L, r, n$ but depends on $\varepsilon, J$. We now let $r \rightarrow \infty$ (with $\varepsilon$ still fixed), obtaining for $M \leq L$,

$$
\begin{gathered}
A_{p}^{\prime} \sum_{|k| \leq M}\left|\sum_{|j| \leq L} f(j) S((1-\varepsilon) k-j)\right|^{p}\left|S\left(\frac{\varepsilon k}{J}\right)\right|^{J p} \\
\quad \leq \frac{1}{1-\varepsilon} \int_{-\infty}^{\infty}|f(t)|^{p} d t+C\left(\sum_{|j| \geq L}|f(j)|\right)^{p} .
\end{gathered}
$$


Next, let $L \rightarrow \infty$, which is permissible as $\sum_{j}|f(j)|<\infty$. We obtain

$$
A_{p}^{\prime} \sum_{|k| \leq M}|f((1-\varepsilon) k)|^{p}\left|S\left(\frac{\varepsilon k}{J}\right)\right|^{J p} \leq \frac{1}{1-\varepsilon} \int_{-\infty}^{\infty}|f(t)|^{p} d t .
$$

We now let $\varepsilon \rightarrow 0+$, and use the continuity of $S$, to obtain

$$
A_{p}^{\prime} \sum_{|k| \leq M}|f(k)|^{p} \leq \int_{-\infty}^{\infty}|f(t)|^{p} d t
$$

Letting $M \rightarrow \infty$ gives

$$
A_{p}^{\prime} \sum_{k=-\infty}^{\infty}|f(k)|^{p} \leq \int_{-\infty}^{\infty}|f(t)|^{p} d t
$$

As $f$ is an arbitrary entire function of exponential type $\leq \pi$, we obtain $A_{p} \geq A_{p}^{\prime}$ and hence $A_{p}=A_{p}^{\prime}$ from earlier results.

Proof of Theorem 1.1. This has been proved above.

Proof of Theorem 1.2. (a) We use a duality argument: if $P$ is a polynomial of degree $<n$, then

$$
\left(\frac{1}{n} \sum_{j=1}^{n}\left|P\left(e^{2 \pi i j / n}\right)\right|^{p}\right)^{1 / p}=\frac{1}{n}\left|\sum_{j=1}^{n} \overline{c_{j}} P\left(e^{2 \pi i j / n}\right)\right|
$$

for some $\left\{c_{j}\right\}$ with $\frac{1}{n} \sum_{j=1}^{n}\left|c_{j}\right|^{q}=1$. Equivalently, if $R$ is a polynomial of degree $\leq n-1$ with $R\left(e^{2 \pi i j / n}\right)=c_{j}$, then for all $j$,

$$
\begin{aligned}
\left(\frac{1}{n} \sum_{j=1}^{n}\left|P\left(e^{2 \pi i j / n}\right)\right|^{p}\right)^{1 / p} & =\frac{1}{n}\left|\sum_{j=1}^{n} \overline{R\left(e^{2 \pi i j / n}\right)} P\left(e^{2 \pi i j / n}\right)\right| \\
& =\left|\int_{0}^{1}(\bar{R} P)\left(e^{2 \pi i t}\right) d t\right|,
\end{aligned}
$$

by the simple sum

$$
\frac{1}{n} \sum_{j=1}^{n}\left(e^{2 \pi i j / n}\right)^{k}=\int_{0}^{1} e^{2 \pi i k t} d t,|k|<n .
$$

We use Hölder's inequality to continue this as

$$
\begin{aligned}
\left(\frac{1}{n} \sum_{j=1}^{n}\left|P\left(e^{2 \pi i j / n}\right)\right|^{p}\right)^{1 / p} & \leq\left(\int_{0}^{1}\left|R\left(e^{2 \pi i t}\right)\right|^{q} d t\right)^{1 / q}\left(\int_{0}^{1}\left|P\left(e^{2 \pi i t}\right)\right|^{p} d t\right)^{1 / p} \\
& \leq\left(B_{q}^{\prime} \frac{1}{n} \sum_{j=1}^{n}\left|R\left(e^{2 \pi i j / n}\right)\right|^{q}\right)^{1 / q}\left(\int_{0}^{1}\left|P\left(e^{2 \pi i t}\right)\right|^{p} d t\right)^{1 / p}
\end{aligned}
$$

by (1.2). As the sum involving $|R|$ is at most 1 , we deduce that

$$
B_{q}^{\prime-p / q} \frac{1}{n} \sum_{j=1}^{n}\left|P\left(e^{2 \pi i j / n}\right)\right|^{p} \leq \int_{0}^{1}\left|P\left(e^{2 \pi i t}\right)\right|^{p} d t .
$$


Thus $B_{q}^{\prime-p / q} \leq A_{p}^{\prime}$, or

$$
B_{q}^{\prime-1 / q} \leq A_{p}^{\prime 1 / p}
$$

Similarly, for some measurable function $g$, with $\int_{0}^{1}\left|g\left(e^{2 \pi i t}\right)\right|^{q} d t \leq 1$,

$$
\left(\int_{0}^{1}\left|P\left(e^{2 \pi i t}\right)\right|^{p} d t\right)^{1 / p}=\int_{0}^{1}(P \bar{g})\left(e^{2 \pi i t}\right) d t=\int_{0}^{1}(P \bar{R})\left(e^{2 \pi i t}\right) d t
$$

where if $g$ has Fourier series $\sum_{j=-\infty}^{\infty} c_{j} e^{i j t}, R(t)=\sum_{j=0}^{n-1} c_{j} e^{i j t}$, and we are using orthogonality. We now again use quadrature on the unit circle, followed by Hölder's inequality, to continue this as

$$
\begin{aligned}
& =\frac{1}{n}\left|\sum_{j=1}^{n} \overline{R\left(e^{2 \pi i j / n}\right)} P\left(e^{2 \pi i j / n}\right)\right| \\
& \leq\left(\frac{1}{n} \sum_{j=1}^{n}\left|R\left(e^{2 \pi i j / n}\right)\right|^{q}\right)^{1 / q}\left(\frac{1}{n} \sum_{j=1}^{n}\left|P\left(e^{2 \pi i j / n}\right)\right|^{p}\right)^{1 / p} \\
& \leq\left(A_{q}^{\prime-1} \int_{0}^{1}\left|R\left(e^{2 \pi i t}\right)\right|^{q} d t\right)^{1 / q}\left(\frac{1}{n} \sum_{j=1}^{n}\left|P\left(e^{2 \pi i j / n}\right)\right|^{p}\right)^{1 / p} \\
& \leq\left(A_{q}^{\prime-1} \sigma_{q} \int_{0}^{1}\left|g\left(e^{2 \pi i t}\right)\right|^{q} d t\right)^{1 / q}\left(\frac{1}{n} \sum_{j=1}^{n}\left|P\left(e^{2 \pi i j / n}\right)\right|^{p}\right)^{1 / p} .
\end{aligned}
$$

In summary,

$$
\int_{0}^{1}\left|P\left(e^{2 \pi i t}\right)\right|^{p} d t \leq\left(A_{q}^{\prime-1} \sigma_{q}\right)^{p / q} \frac{1}{n} \sum_{j=1}^{n}\left|P\left(e^{2 \pi i j / n}\right)\right|^{p} .
$$

Thus $B_{p}^{\prime} \leq\left(A_{q}^{\prime-1} \sigma_{q}\right)^{p / q}$, or swapping the roles of $p, q, B_{q}^{\prime} \leq\left(A_{p}^{\prime-1} \sigma_{p}\right)^{q / p}$. Hence,

$$
\left(B_{q}^{\prime 1 / q}\right)^{-1} \sigma_{p}^{1 / p} \geq\left(A_{p}^{\prime}\right)^{1 / p} .
$$

This, (2.9), and Theorem 1.1 give (1.6).

(b) Fix $n$ and let $\ell_{0 n}$ be as in (2.4). For $s \in[0,1]$ and $p>0$, let

$$
\chi_{p}(s)=\int_{0}^{1}\left|\ell_{0 n}\left(e^{2 \pi i(t-s)}\right)\right|^{p} d t-\frac{1}{n} \sum_{j=0}^{n-1}\left|\ell_{0 n}\left(e^{2 \pi i(j / n-s)}\right)\right|^{p}
$$

denote the quadrature error for the polynomial $\ell_{0 n}\left(z e^{-2 \pi i s}\right)$. Observe that

$$
\chi_{p}(0)=\int_{0}^{1}\left|\ell_{0 n}\left(e^{2 \pi i t}\right)\right|^{p} d t-\frac{1}{n}
$$

and, in particular, $\chi_{2}(0)=0$ by (2.8). Inasmuch as $\left|\ell_{0 n}(z)\right|<1$ unless $z=1$, this shows that

$$
\chi_{p}(0) \begin{cases}>0, & 0<p<2 \\ <0, & p>2\end{cases}
$$


Thus for $p \neq 2, \chi_{p}(s)$ is a continuous, not identically vanishing function of $s$. Also by periodicity,

$$
\int_{0}^{1} \chi_{p}(s) d s=0 .
$$

It then follows that for some values of $s, \chi_{p}(s)>0$, and for others, $\chi_{p}(s)<0$. The former inequality shows that $B_{p}>1$, and the latter $A_{p}<1$.

\section{REFERENCES}

[1] Ralph Philip Boas Jr., Entire functions, Academic Press Inc., New York, 1954. MR0068627 $(16,914 \mathrm{f})$

[2] D. P. Dryanov, M. A. Qazi, and Q. I. Rahman, Entire functions of exponential type in approximation theory, Constructive theory of functions, DARBA, Sofia, 2003, pp. 86-135. MR2092333(2006a:41008)

[3] Carolyn Eoff, The discrete nature of the Paley-Wiener spaces, Proc. Amer. Math. Soc. 123 (1995), no. 2, 505-512, DOI 10.2307/2160908. MR.1219724 (95c:42011)

[4] F. Filbir and H. N. Mhaskar, Marcinkiewicz-Zygmund measures on manifolds, J. Complexity 27 (2011), no. 6, 568-596, DOI 10.1016/j.jco.2011.03.002. MR 2846706

[5] B. Ya. Levin, Lectures on entire functions, Translations of Mathematical Monographs, vol. 150, American Mathematical Society, Providence, RI, 1996. In collaboration with and with a preface by Yu. Lyubarskii, M. Sodin and V. Tkachenko; Translated from the Russian manuscript by Tkachenko. MR.1400006 (97j:30001)

[6] Eli Levin, D. S. Lubinsky, $L_{p}$ Christoffel functions, $L_{p}$ universality, and Paley-Wiener spaces, to appear in J. d'Analyse de Mathematique.

[7] D. S. Lubinsky, Marcinkiewicz-Zygmund inequalities: methods and results, Recent progress in inequalities (Niš, 1996), Math. Appl., vol. 430, Kluwer Acad. Publ., Dordrecht, 1998, pp. 213-240. MR.1609947(99a:41045)

[8] D. S. Lubinsky, A survey of weighted polynomial approximation with exponential weights, Surv. Approx. Theory 3 (2007), 1-105. MR2276420 (2007k:41001)

[9] M. Plancherel and G. Pólya, Fonctions entières et intégrales de fourier multiples (French), Comment. Math. Helv. 10 (1937), no. 1, 110-163, DOI 10.1007/BF01214286. MR1509570

[10] A. F. Timan, Theory of approximation of functions of a real variable, Dover Publications Inc., New York, 1994. Translated from the Russian by J. Berry; Translation edited and with a preface by J. Cossar; Reprint of the 1963 English translation. MR.1262128 (94j:41001)

[11] A. Zygmund, Trigonometric series. Vol. I, II, Cambridge Mathematical Library, Cambridge University Press, Cambridge, 1988. Reprint of the 1979 edition. MR.933759 (89c:42001)

School of Mathematics, Georgia Institute of Technology, Atlanta, Georgia 30332 0160

E-mail address: lubinsky@math.gatech.edu 